\title{
A DISJUNCTIVE ANALYSIS OF NEONATAL APPROACH STIMULUS PREPOTENCE,
}

\author{
by \\ Stephen E. Sigman \\ Thesis submitted to the Graduate Faculty of the \\ Virginfa Polytechnic Institute and State University \\ In partial fulfillment of the requirements for the degree of \\ Master of Science \\ In \\ Psychology
}

APPROVED :

A. H. Schulman, Cha1rman

R. W. Cravens

A. A. Spevack

June, 1974

Blacksburg, Virglnia 


\section{ACKNOWLEDGEMENTS}

The problem was suggested to the author by

- The

author is indebted to

for his invaluable advice and criti-

cisms during the execution of the experiment and during the preparation of this paper.

Special thanks to

for directing my thinking by asking those poignant questions. 
TABLE OF CONTENTS

\section{Page}

Acknowledgements

11

List of Tables

iv

List of Figures

v

Introduction

1

Visual stimuli

Auditory stimuli

Compound stimuli: Visual-plus-Auditory

Method

Subjects

14

Apparatus

14

Procedure

17

Results

Incidental Observations

20

Approach data

Following data

29

Discussion

35

Sumnary and Conclusions

40

References

41

Vita

45 


\section{TABLE}

Page

1. Summary of results obtained with four groups of chicks in two approach and one following trial. 


\section{FIGURES}

Page

Figure 1. The apparatus.

Figure 2. Approach latencles for those chicks exposed to the compound stimulus on trials one and two.

Figure 3. Trial two approach latencies for the $\mathrm{L}, \mathrm{T}$, and $L \& T$ groups at 24 and 36 hours.

Figure 4. Trial two approach latencies for the L, T, and $\mathrm{L} \& \mathrm{~T}$ groups at 24 and 36 hours; nonresponders not included.

Figure 5. Number of chicks approaching in the $\mathrm{L} \& \mathrm{~T}, \mathrm{~T}$, and $L$ groups at 24 and 36 hours.

Figure 6. Number of chicks following in the $L \& T, T$, and L groups at 24 and 36 hours.

Figure 7. Number of chicks following the light and number of chicks following the tone at each age in the disjunctive ( $\mathrm{L}$ vs. $\mathrm{T}$ ) group.

Figure 8. Comparison of the number of chicks following the light (A) in the $L$ vs. $T$ and the $L$ groups, and the number of chicks following the tone (B) in the $\mathrm{L}$ vs. $\mathrm{T}$ and the $\mathrm{T}$ groups. 


\section{INTRODUCTION}

The term imprinting was coined by Lorenz (1937) to refer to the special process by which neonates of nidifugous species of birds form long-lasting social attachments to their parents or other objects. The fact is that these neonates often approach and follow the first moving object to which they are exposed. Lorenz (1935) has shown that after exposure to a certain object, young birds will subsequently follow only that object, or class of objects. This process has been demonstrated to be confined to a brief period in the bird's life, and once the attachment is established it is often very stable (Sluckin, 1973, p.13). Moreover, the attachment to the imprinted object is critical in the development of later behaviors such as courtship and mating. Since Lorenz's revival of the interest in Imprinting, Investigators have experimentally investigated many aspects of this phenomenon, including the relative permanence of the attachment, the reversibility of the attachment, the "critical period" In which the attachment is formed, and so forth.

One focal point of the more recent research on the phenomenon of imprinting has been the determination of the specific stimulus properties of an object which directs the approach and following response (1.e., Fischer, 1966; Gottlieb \& Klopfer, 1962; Smith, 1960). Although the role of the stimulus as either a relnforcer for the approach response (Moltz, 1960) or as a releaser of the response (Hess, 1973) is not clear, the irrefutable conclusion of a plethora of reports is clear: 
different types of stimull have varying effectiveness in promoting the occurrence of approach and following behavior (Hess, p. 87). Some investigation of the role of tactile and thermal stimulation in the approach response has been done (1.e., Smith \& Bird, 1963), but the great majority of the research reported in this area has centered on the relative importance of visual and/or auditory properties of the imprinting stimulus.

\section{Visual Stimuli}

As Lorenz (1935) has reported, certain specles of birds can and will become imprinted on a variety of animate or inanimate objects. Though it has been demonstrated that neonatal chicks can develop a preference for familiar, stationary objects as opposed to unfamiliar stationary objects (1.e., Gray, 1960) the fact that moving objects are superior to stationary objects as imprinting stimuli is acknowledged by virtually all investigators. Indeed, one of the first investigators to treat the phenomena of imprinting in a scientific manner (Spalding, 1873) reported that newly hatched chicks would approach the first moving object to which they were exposed. Among the moving objects which have been successfully employed in elictting neonatal approach and following are green cubes (Jaynes, 1957), decoys (Ramsey \& Hess, 1954), boxes and balloons (Fabricius, 1955). Hinde, Thorpe and Vince (1956) have reported that young moorhens and coots will follow a wide variety of objects. Furthermore, they report that the same birds will approach and follow other, similar objects in later trials, but only if the objects are moving. Humans also can serve as adequate imprinting stimuli as 
witnessed by Fabricius' (1951) report that when he walked away from ducklings they readily followed him; if he remained motionless, however, the ducklings would not approach him and they appeared to ignore him completely.

While it appears that almost any moving visual stimulus can direct the approach and following responses in at least some precocial neonates, certain stimuli are more effective than others. A very wide range of stimulus sizes has been found to suffice (Fabrictus \& Boyd, 1954) but Investigators have found, for example, that chicks 14 to 22 hours old approach red cardboard disks 10 to $20 \mathrm{~cm}$. in diameter in greater proportion than smaller or larger diameters (Schulman, Hale and Graves, 1970). Similarly, irregular movement has been reported to be more effective in directing approach than regular movement (Salzen \& Sluckin, 1959) and retreating objects produce more following than approaching or stationary objects (Moltz, 1963). Schulman, et. al. (1970) falled to confirm Moltz's finding, however.

Observations by Menner (see James, 1959 and Moltz, 1960, p. 304) have suggested a possible explanation for the sensitivity of nidifugous neonates to movement. Menner has noted that, due to the peculiar structure of the pecten, the fluctuation of retinal 11lumination which a moving object normally produces is enhanced in the avian eye. The pecten, a conical structure which projects from the blind spot of the eye towards the pup11, casts shadows on the retina which Increases the amount of retinal flicker produced by a moving object. It has been proposed that it is this.fluctuation in 1llumination that the bird first responds to in the Imprinting situation (James, 1959, p. 59). 
Largely because of these observations, several investigators have examined the development of the approach response to simulated movement. Smith (1960) has demonstrated that visual stimulation which includes an intermittant element is sufficient to elicit the approach response upon initial exposure in 16 to 26 hour old chicks of the domestic fowl. Chicks approached a 12 inch, rotating white disc on which a $45^{\circ}$ black sector had been painted, but they did not approach an identical disc which did not rotate, but which approached and retreated from the chick. Citing Menner's theory of the effects of the pecten in the avian eye, James (1959) has investigated the effectiveness of several different rates of flicker in eliciting the initial approach response in Plymouth Rock chicks. Chicks were exposed at 48 hours of age to lights which flickered at an on-off rate of either $.25 / .25 \mathrm{sec}, 1.0 / 1.0 \mathrm{sec}$. or $5.0 / 5.0$ secs. Though these rates did not differ significantly in effectiveness, the conclusion that the rate of flicker can be varied over a relatively wide range without appreclable effects must be questioned in view of the confounding of rate of flicker and the duration of each light pulse in this study. In another part of the same experiment, James paired a flickering light (on-off rate: $.25 / .25$ sec.) with a stationary polythene ball and found that chicks subsequently followed the ball in the absence of any flickering 1ight. In summarizing his results, James concluded that "flicker constitutes an adequate unconditioned stimulus for approach behavior" (James, 1959, p. 66).

A subsequent report by James (1960) has supported the notion of flicker as UCS for the approach response. In this experiment James exposed chicks either one day old or seven days old to a stationary 
beaker which was paired with a flickering light. Subsequent tests of approach to or withdrawal from the moving beaker alone resulted in signiflcantly less approaching in those initially exposed at 7 days than those exposed at one day of age. This suggests that, as is the case with more traditional moving imprinting stimuli, the attractiveness of visual flicker decreases with age, at least over the age range tested. In addition, an on-off rate of $.2 / .2$ secs. was more effective than an on-off rate of $.2 / 5$ secs.

Smith \& Hoyse (1961) have reported a series of systematic experiments in which a variety of intermittant visual stimuli were used successfully to elicit approach upon Initial exposure to the stimulus. Using 18 to 30 hour old chlcks of domestic fowl they found, among other things, that the diameter of a flickering light, intensity of the light and the angle of regard of the light is critical in the elicttation of the approach response. They report also that there were no significant differences in proportion of birds approaching to elther a red, green or white 1ight. Intensity has been shown to be a roughly U-shaped function by Kovach $(1971 \mathrm{a}, \mathrm{b})$ but color has also been shown to be important, maximal approach occurring to a blue light (Kovach \& Hickox, 1971). Simner (1966) and Gottlieb \& Simner (1969) have found a flicker rate of $3 \pm 1$ pps to be maximally effective in eliciting the approach response in chicks. Previous publications suggested that the capacity of a visual stimulus to elfctt the approach response increased with age, due to the maturation of the visual system (cf. Gott1leb, 1963). These studies will be considered later in a slightly different context, but it should be noted that Kovach, Paden \& Wilson (1968) have reported 
that approach to a light flickering at 3.5 pps is age dependent; a marked increase in approach responses was found in birds tested at 12 , 24 and 72 hours.

\section{Auditory Stimuli}

Just as intermittent visual stimuli have been shown to elicit approach and following responses, intermittant auditory stimuli have also been shown to suffice as elicitors of these responses (1.e. Collias \& Collias, 1956; Fabricius, 1951). Collias (1952) found low frequency tones to elfcit the approach response in young chicks, though Fischer (1972) has suggested that frequency of the stimulus is not of major import in tones of very short duration. Boyd \& Fabricius (1965) found that by standing outside one end of a runway and repeating "kom-kom" they could induce naive mallard ducklings to approach that end of the runway. Further, by quickly alternating ends at which the experimenter stood, the ducklings could be induced to "follow" the voice, moving from one end of the apparatus to the other. Ducklings, then, are able to localize a source of sound, even when that source is not visible. Salzen \& Sluckin found that chicks, too, are able to accurately localize a sound source, even when that source is hidden behind a screen (Sluckin, 1973, p. 29).

Many rhythmic, repetitive auditory stimuli have been shown to be sufficient to induce following in several species of newly hatched ducks, though as is the case with visual stimull, some are more attractive than others (Klopfer, 1959). Indeed, preferences for certain auditory stimuli over others have been reported by several investigators. 
For Instance, Collias \& Collias (1956) placed several species of 24 hour old ducklings in a runway, from one end of which emanated either the attraction call or the distress call of the parent species. They report that the ducklings approached the attraction call and avolded the distress call. More recently Gottlieb (1965) has reported an Innate preference (as measured by proportion of subjects following and latency to respond) for the species-specific maternal call among 24 hour old mallards. Similar preferences have been reported for White Rock chicks (Gott1leb, 1966).

Fischer (1972) has systematically varled the physlcal properties of tones which elicit following in young domestic fowl. Though she found no exclusive preferences, optimal following occurred to very brief, repetitive tones with a frequency of about $500 \mathrm{~Hz}$.

\section{Compound stimuli: Visua1-plus-Auditory}

That intermittant auditory and visual stimuli are both suffictent to elicit approach and following responses is, then, well established. However, in a natural setting wherein young birds imprint most probably on the parent of their species, they are exposed to a "total" imprinting object, i.e., one which has both auditory and visual properties. The question then arises as to which of the two modalities plays the dominant role in the imprinting situation.

Several investigators have attacked this question directly and it has been generally reported that visual otfmull are more effective when they emit sound than when they are silent (Hess, 1973, p. 87). For example, Smith \& Bird (1963) have reported that 24 hours old chicks 
are more likely to approach a combination of auditory and visual stimuli than either of the stimuli alone. Similarly, Gottlieb (1968) exposed 16 to 30 hour old Wood ducks and Mallards to a vocal mode1, a silent model, or a non-visible moving vocalization alone. He found the vocalization alone to be more effective in eliciting following than the silent model alone. However, the vocalization and model in combination was more effective than either of the two stimuli alone.

This finding indicates that the importance of auditory stimuli alone, or the importance of visual stimuli alone in imprinting is not absolute, but relative to the other stimulus' effectiveness. The fact that an auditory stimulus alone, or a visual stimulus alone is not as effective as a combination of the two stimuli is what led Gottlieb to refer to "the fallure of the purely auditory stimulus to fully meet the stimulative requirements of the chick ..." and to state that "it seems 1ikely that the combination of auditory and visual stimuli emanating from a single source is required to maximally sustain the chick's approach response" (Gottlieb \& Simner, 1969, p. 62).

The question of the relative importance of auditory and visual stimuli in the imprinting situation has been investlgated by Klopfer \& Gottlieb (1962). They exposed 10 to 20 hour old Pekin ducklings to a vocal mallard duck decoy as it traversed a circular runway. At approximately 30 hours of age the ducklings were again placed in the runway where they were exposed to efther the silent moving decoy or the vocal1zation which emanated from a statlonary speaker. Approach and followIng scores indicated that a developmental variable was operating; those birds exposed Initially during the first half of their critical perlod 
(day 27 after incubation onset) responded only to the auditory stimulus, while those exposed during the second half of their critical period responded to visual as well as auditory stimuli. It was suggested that further maturation of the visual system during the critical period was responsible for the emergence of this visual tendency, and this tendency should come to equal or surpass the auditory tendency.

One hypothesis that has arisen from the research on the auditory vs. visual issue has been that of behavioral polymorphism (Klopfer \& Gottlieb, 1962). These Investigators noted that in any given clutch of ducklings, some Individuals show tendencies to approach visual stimuli, some show tendencies for auditory stimuli, and others show no selective tendencies at all. It has been suggested that these individual differences between the auditory preference and visual preference ducklings represents a behavioral polymorphism (Klopfer \& Gottlieb, 1962). It is argued that these different response tendencies in the individuals ensure, through social facilitation, the development of the same response tendency in the entire group thus accounting for the behavioral synchrony a clutch of ducklings achieves (see Klopfer, 1959). Thus, despite the different developmental ages of the individuals of a clutch, they will all respond to the same imprinting stimulus, typically the female parent.

Some evidence for the behavioral polymorphism hypothesis was obtained in Klopfer \& Gottlieb's (1962) report. There was no signif1cant correlation between the strength of the responses to the auditory and visual stimuli; this was interpreted as support for the polymorphism hypothesis (Klopfer \& Gottlieb, 1962). 
In a study directed specifically at the issue of the emergence of visual Imprinting with age, Gottlieb \& Klopfer (1962) have partially replicated their earlier finding. A simflar procedure was used; Pekin ducklings 6 to 31 hours of age (posthatch) were exposed to a vocalizing mallard decoy as it traversed a circular runway, and the ducklings were exposed 10 to 14 hours later to the silent model alone, and then to the vocalization alone. Half the birds were reared in isolation and half were reared communally. Isolate birds were found to show a greater amount of auditory imprinting during the first half of their critical period and a greater amount of visual imprinting in the second half of their critical period.

The two studies presented thus far (Klopfer \& Gottlleb, 1962; Gottlieb \& Klopfer 1962) have had a common procedure, 1.e., the ducklings were Inftially exposed at one age, and tested at a later age. As Hess (1973) has pointed out, such a procedure may not be appropriate for determining the preference of a duckling upon initial exposure. In other words, a test trial 10 to 14 hours after initial exposure may not necessarily reflect the duckling's preference at the initial exposure.

This procedural difficulty was corrected in a study done by Gottlieb in 1963. Pekin ducklings were tested as to preference on their initial exposure (as measured by latency to respond and proportion of subjects responding). Gott1leb reported that "the duckling's ability to follow a model sheerly on the basis of visual stimulation increases with age and that auditory stimulation plays an important activating or attentiondirecting function at all ages" (Gottlieb, 1963). His ducklings preferred a vocal, moving model to a silent moving model throughout their 
entire critical period (day 27 to day 28-1/2 after incubation onset). Visual response tendencies were much lower than previously reported tendencies, and auditory tendencies were considerably higher. Thus, Hess (1972, pp. 95-96) argues "1t is therefore very apparent that there are changes in the relative responsiveness of Pekin ducklings to visual and auditory stimuli with age. The responsiveness to stimulation which is present at one age cannot necessarily be determined by tests administered at another age. Nevertheless, the main conclusion made by Gottlieb \& Klopfer (1962) --that auditory responsiveness appears predominant before the developmental age of 27-1/2 days from incubation--still holds. However, the initial postulation--that visual responsiveness in chicks past that age--obviously does not. The observed increase in visual responsiveness observed by Gottlieb \& Klopfer (1962) must therefore be a function of the later test age which these authors utilized."

Gottlieb (1968) presented 16 to 30 hour old Mallard and Wood duck ducklings with a simultaneous choice situation involving a silent moving duck decoy and a non-visible moving maternal call of their species. The ducklings were placed in a circular runway around which moved, on the inside, a silent decoy; the speaker from which the call emanated was $180^{\circ}$ opposite the decoy, on the outside of the runway, and rotated as the decoy rotated. Under these conditions none of the ducklings followed the visual stimulus at all; both species responded promptly to the call, though neither accumulated a high following score due to lagging behind or, when close, attempting to jump the wall to approach the call Gottlieb, 1968). 
It appears, then, that ducklings respond primarlly to auditory stimuli in the imprinting situation, though this preference is probably best not described as imprinting (see Gottlieb, 1965 and Gottlieb, 1968).

Fischer (1966) has presented evidence that day old chicks are primarily auditory imprinters. Twenty-four hour old White Giant x P1lch broiler chicks were initially exposed to a moving, vocal model. The chicks were retested at 36 hours to the model with the same, different or no auditory stimulus, or a different model with the same, different or no auditory stimulus. The original auditory stimulus produced significantly more following (as measured by time spent following) than the different auditory stimulus with the same model. Even the different auditory stimulus produced significantly greater following than no auditory stimulus at all. In addition, one group of chicks initially exposed to the silent moving model showed "negligible" following when retested on the same silent model (Fischer, 1966). Again, because the chicks were tested at a later age after initial exposure, inferences about the relative attractiveness of the stimuli at the inftial exposure are best made with caution.

One recent study with White Rock chicks by Gottlieb \& Simner (1969) has measured preference in a simultaneous choice situation upon Initial presentation. The stimuli, a flickering light and an intermittant "popping" were previously matched as to attractiveness to nalve chicks. In a runway situation with the light at one end and the "pop" at the other, latency and first choice scores revealed a preference for the auditory stimulus at both ages tested (20 and 40 hours) supporting the 
hypothesis that auditory stimulation is relatively more important than visual stimulation, at least in the first few days after hatching. These results are in accord with the data previously reported by Gottlieb (1968) and do not support the behavioral polymorphism hypothesis.

It can be seen that, in the more recent literature on the subject, a consensus has developed that precocial neonates respond primarily to auditory stimulation during the first few days of life. However, all of these studies have had a similar procedure. Inftially subjects were presented with a compound.stimulus and then tested on either one or the other of its components, or they were given a choice between the two separated stimuli. In effect, then, the experimenter made the choice for the subject. Especially in light of the evidence that the best approach to objects and the most sustained following occurs when the auditory and visual stimuli are presented together (Gottlieb \& Simner, 1969) it would seem desirable to test the subjects on the compound stimuli rather than on 1 ts component parts, and to allow the subject the active role in the choice situation. With the prediction that auditory stimuli will be prepotent over visual stimuli in elfciting approach and following behavior upon inttial exposure, the present study was undertaken to examine the possibility that this auditory prepotence is a result of the test procedure utilized in previous studies. To this end a stimulus disjunction procedure was developed which provided the subject with a unique simultaneous choice situation. 
METHOD

Subjects: Eggs of the Canadian Athens random bred strain of chickens were acquired on a weekly basis from the Virginia Polytechnic Institute and State University Poultry Science Department and were incubated in a forced-air incubator-hatcher maintained at $37.5^{\circ} \mathrm{C}$ and $65 \%$ relative humidity. During hatching the incubator was checked at regular intervals and the chicks were removed as soon as they emerged from the shell and placed in isolation cages located in the incubator room. The cages, which provided visual but not auditory 1 solation, were $18 \mathrm{~cm}$ wide, $24 \mathrm{~cm}$ long, and $18 \mathrm{~cm}$ deep. Temperature in the incubator room was maintained at $90^{\circ} \mathrm{F}$. ; constant 1 llumination was provided by overhead flourescent lights. An equal number of chicks from each weekly batch were assigned to each of the four treatment conditions unt11 a total of 72 subjects had been successfully tested.

Apparatus: The apparatus, depicted in Figure 1, consisted of a modified straight alley runway $90 \mathrm{~cm}$ long, $15 \mathrm{~cm}$ wide and $25 \mathrm{~cm}$ deep, constructed of unpainted plywood. A $10 \mathrm{~cm} \mathrm{X} 10 \mathrm{~cm}$ X $25 \mathrm{~cm}$ start box was located midway along one of the outside walls; a sliding plywood door allowed access to the runway. The entire wall of the runway opposite the start box constituted a projection screen formed by a plywood frame covered with translucent brown paper and, on the inside, translucent brown plastic. An "approach" area, $7.5 \mathrm{~cm} \times 10 \mathrm{~cm}$ was Inscribed in ink on the runway floor opposite the start box, next to the projection wall. 


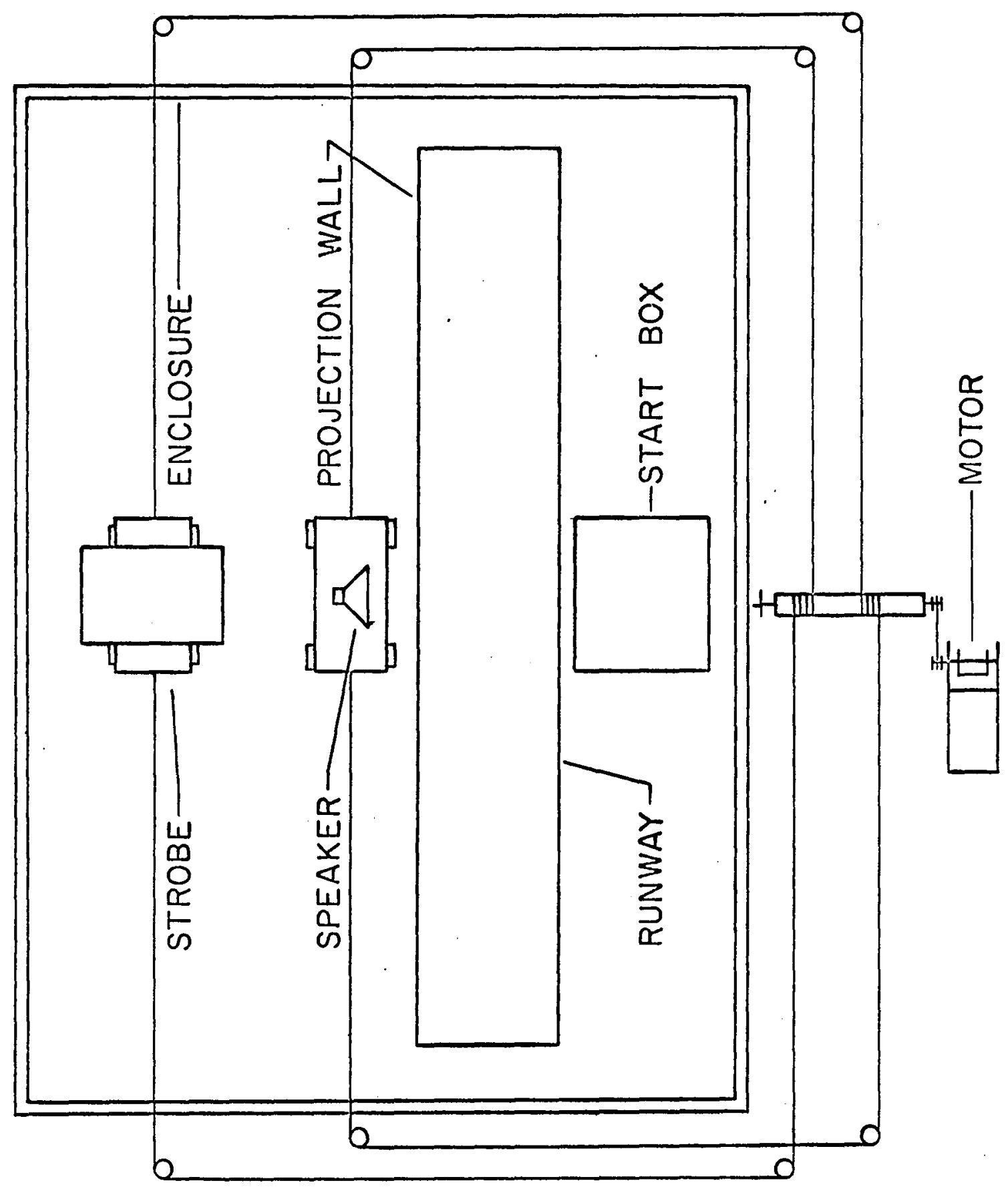

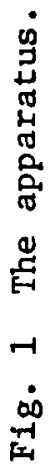


Behind the projection wall were located two small carts, each mounted on a separate track running parallel to the runway. A serles of cords and pulleys made it possible to pull each cart the entire length of the runway, in either direction, in unison or in opposition. Power to propel the carts was provided by an $A 47$ G1lbert electric motor. On the cart nearest the runway wall was mounted a speaker through which the auditory stimulus was presented. A small stroboscopic light which projected its beam onto the projection wall of the runway was mounted on the other cart to provide the visual stimulus. The strobe light was enclosed in an insulated box to eliminate the otherwise audible pop which accompanied each light pulse.

The runway, carts and mountings were placed inside a sound attenuated plywood box. It was possible to observe the inside of the box through two plexiglass windows located in the top of the box, directly over the runway. The entire apparatus was located in a test room separate from the incubation and housing room. Illumination of the test room was provided by a single 100 watt bulb suspended 3 feet over the top of the apparatus. Temperature in the test room was maintained at $74^{\circ} \mathrm{F}$ throughout the experiment.

The auditory stimulus, found by Fischer (1973) to be maximally attractive to naive chicks, consisted of a pulsing $500 \mathrm{~Hz}$ tone; the pulse rate was 4 per second, with a pulse duration of $50 \mathrm{msec}$. The tone intensity was $60 \mathrm{db}$ as measured from Inside the start box. The parameters of the visual stimulus were those reported by other researchers (Gottlieb \& Simner, 1969; Kovach, 1970; Smith, 1960) to be maximally attractive to naive chicks. The visual stimulus, as it appeared on the 
inside runway wall, was an almost circular, white 1 mage $12.5 \mathrm{~cm}$ in diameter and $5 \mathrm{~cm}$ from the floor of the apparatus. The strobe was adfusted to a flash rate of 3.5 pps. As measured on the Inside wall of the runway, the illuminance of the visual stimulus was .8 feet candles, A pilot study confirmed that the two stimuli were equally attractive to naive chicks. An approach rate of $20 \%$ was obtalned to both stimuli at 24 and 36 hours. These approach rates are less than those reported by other investigators using simflar stimuli (Gottlleb \& Simner, 1969) but there are differences in procedure between those studies and the present study, such as the length of time allowed for a response to be made. Moreover, there are differences in the strain of chickens employed. Nevertheless, since attractiveness changes over time (Sluckin, 1973, Chapt. 2), an increased rate of responding would be expected with exposure to the stimuli.

Procedure: Subjects were assigned to one of four stimulus conditions ( $n=18$ per condition): 1) light and tone moving in opposition ( $\mathrm{L}$ vs. T), 2) light and tone moving in unison ( $L \& T$ ), 3) light (L) and 4) tone (T). Nine subjects from each group were tested at 24 hours posthatch and nine were tested at 36 hours posthatch.

Each chick was carried from the incubator room to the test room in a small cardboard box and placed in the start box of the apparatus. Sixty seconds later the start box door was opened and the stimull, both located directly opposite the start box, were turned on for the familLarization period. A wire screen placed In front of the 6 tart box doorway prevented subjects from approaching during familiarization. The stimuli remained motionless for the first 3.5 minutes of familiarization, 
then they traversed in unison the entire length of the runway for 1 minute. After the minute of movement the stimuli were returned to the starting place directly opposite the start box, where they remained motionless unt1l the end of the familiarization pertod. At the end of familiarization, the stimuli were turned off, the start box door was closed, and the wire screen was removed.

Sixty seconds later the stimuli were turned on and the start box door was opened for the first approach trial. Subjects were allowed 3 minutes in which to stop inside the rectangular approach area while orfented towards the projection wall, defining an approach response. Latency to approach was recorded. Those subjects which falled to approach within 3 minutes were discarded. Those subjects which did approach were allowed 30 seconds in the presence of the stimuli; the stimul1 were then turned off, the subject was returned to the start box, and the door was closed.

Sixty seconds later the start box door again was opened to begin the second approach trial. Concurrent with the opening of the start box door, the appropriate stimulus or stimuli were turned on. For nine subjects in each age group the appropriate stimulus was fust the light, for nine subjects it was just the tone, and for 18 subjects 1 was light and tone. Once again, 3 minutes was allowed for the approach response to be made and latency to approach was recorded. Those subjects which did not approach were recorded as "no approach, no follow". For those subjects which did approach, the electric motor was engaged as soon as the response was made and the stimulus or stimuli moved to the left or right end of the runway. For nine of the $L \& T$ group the stimuli moved 
In unison, and for the other nine the stimuli moved in opposite directions. Subjects were recorded as having followed a stimulus if they exited the approach area in the same direction in which the stimulus moved. The direction in which the stimuli moved was varied randomly from subject to subject. If a subject had not left the approach area by the time the stimull reached the end of the runway, about 25 seconds, the subject was recorded as "no follow". 
RESULTS

Results, Including percentage approach, latency and number of approaches, and number of followers in each condition are summarized in Table 1 .

For the purpose of statistical analysis, subjects who did not approach on trial two were assigned the maximum score (180 sec.). In those comparisons involving a parametric Analysis of Variance, a root transformation $(\sqrt{x} \& \sqrt{X+1})$ was performed on the raw scores to satisfy the assumptions of homogeneity of varfance (Fischer, 1969).

\section{Incidental Observations}

Calls---Subject's vocalizations, when first placed in the start box and often until familiarization was over, were characterlzed primarily by the relatively loud cheeps, descending in pitch, generally known as distress calls (Slucken, 1973, p. 16). Just prior to approaching a particular stimulus, the chick's call generally became the rather high, twittering notes known as contentment calls (Sluckin, p. 17). The chicks also emitted contentment calls while following the stimuli, and no difference in the stimuli in this regard was noted, though these observations were not systematic. When the stimuli were turned off, especially at the end of trial one, but also at the end of trial two and the following trial, the chlcks vocalizations changed again to the distress calls.

Approach---Typically, when approaching a stimulus the chicks would exit the start box and pause for a few seconds fust outside the approach 


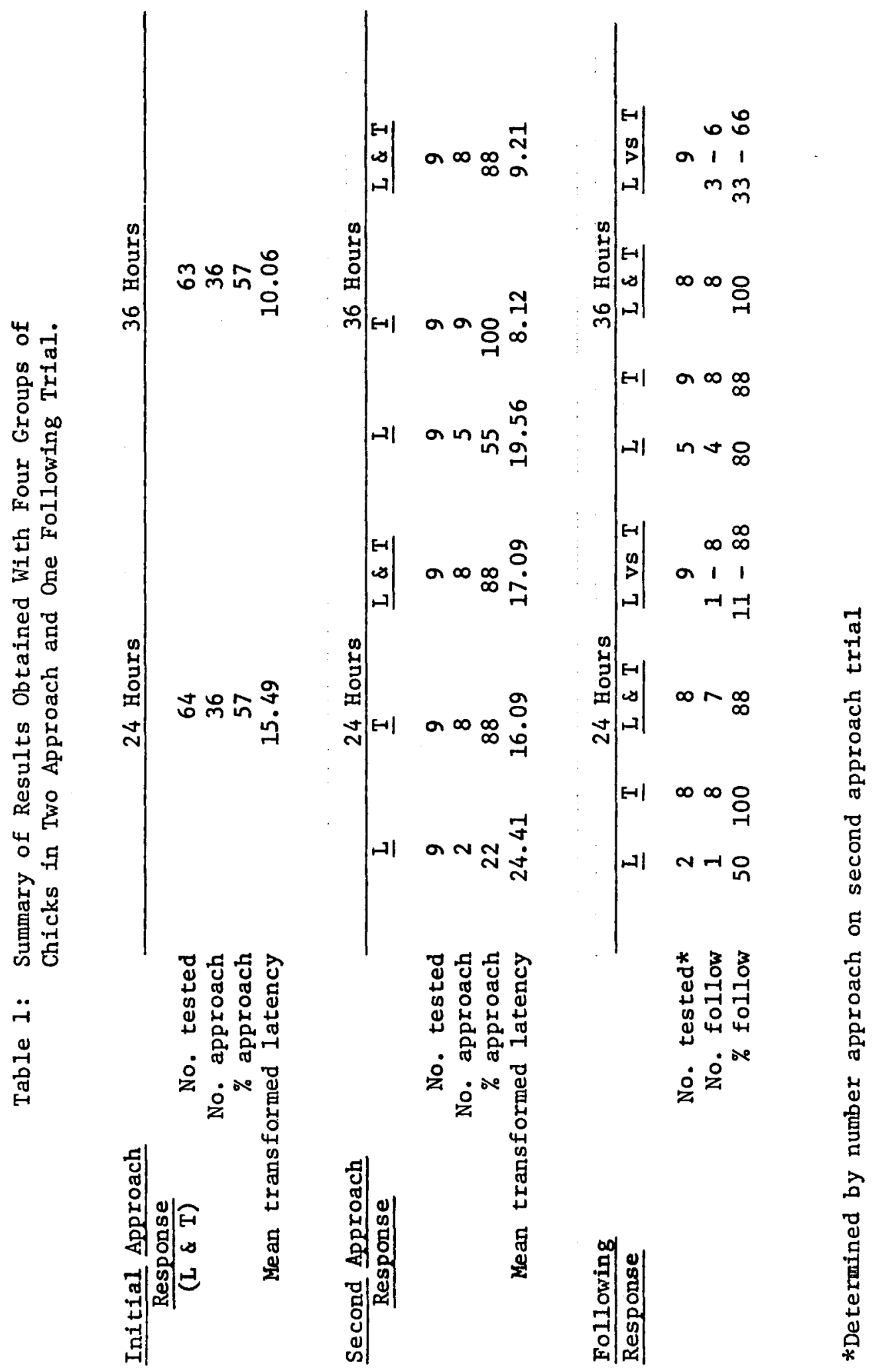


area before finally approaching the stimulus, often in a sudden leap. Almost without exception, those subjects which were recorded as "no approach" failed to leave the start box.

Following---A few chicks which were exposed to the stimulus disjunction vacillated between the two parting stimuli, moving a step or two in one direction, then rapidly shifting directions, and so forth. The chicks which followed the light alone or the tone alone did not show any vacillation; their following was rapid, a smooth transition from approaching the stimulus to following. In following, the chicks typically pressed their bodies firmly up against the projection wall and sometimes fumped as if trying to get close to the stimulus. Those subjects which approached but were recorded as "no follow" almost without exception remained in the approach area. A few subjects which responded to elther the light alone or the tone alone by approaching and following were, after that test trial, exposed to the other stimulus. Most chicks given such a confirmation trial were consistant in their choice; if they approached the tone alone, they did not approach the light alone. One subject which initially approached and followed the light did, however, approach and follow the tone on the subsequent confirmation trial.

\section{Approach data}

On trial one, wherein all subjects were allowed the opportunity to approach I \& $\mathrm{T}$, an approach rate of $57 \%$ was obtalned for both ages. On trial two, wherein subjects were exposed only to their group's specifled stimulus or stimuli, $22 \%$ of the 24 hour subjects exposed to L approached. 
At 36 hours, this increased to $55 \%$ approaching $L$. In the $T$ and the $L$ \& $\mathrm{T}$ groups, $88 \%$ approach was obtained at 24 hours. At 36 hours, this remained the same for the $L \& \mathrm{~T}$ group, but increased to $100 \%$ for $\mathrm{T}$. The mean transformed approach latencies for trials one and two are presented in Figure 2. Only those subjects which were presented with the compound $\mathrm{L} \& \mathrm{~T}$ stimulus at the beginning of both trials, 1.e., those In the $L \& T$ and the $L$ vs. $T$ groups, were included in the calculation of these group means. Statistical analysis failed to demonstrate a significant difference between trials one and two $(F=1.49, \mathrm{df}=1 / 34, \mathrm{p}>.05)$, but the age difference which is evident in the figure is significant, 36 hour old chicks approaching faster than 24 hour old chicks $(F=15.25$, $d f=1 / 34, p<.01)$. The Age $x$ Trial interaction was not significant $(\mathrm{F}<1)$.

The mean transformed approach latencies for the $\mathrm{L}, \mathrm{T}$, and the $\mathrm{L} \& \mathrm{~T}$ groups on trial 2 is presented in Figure 3. It can be seen that in each stimulus condition the age effect noted above is operating, 36 hour old chicks approaching sooner than 24 hour old chicks. In addition, the $L$ group is seen to have approached slower than either the $T$ or $L \& T$ groups, at both ages. Since there were a large number of nonresponders given maximum 180 sec. scores in the $L$ condition (see below) a distribution-free analysis of variance test was used in the analysis of those data (Wilson, 1956). The main effect for age was significant $\left(x^{2}=5.67\right.$, $p<.02)$, as was the maln effect for condition $\left(x^{2}=8.36, p<.02\right)$. Approach latencies to $T$ and $L \& T$ do not differ significantly, either at 24 hours $(U=37.5, p>.05)$ or at 36 hours $(U=23, p>.05)$, but approach latencies to $L$ and $L \& T$ do differ at 24 hours $(U=17, P(.02)$ and at 


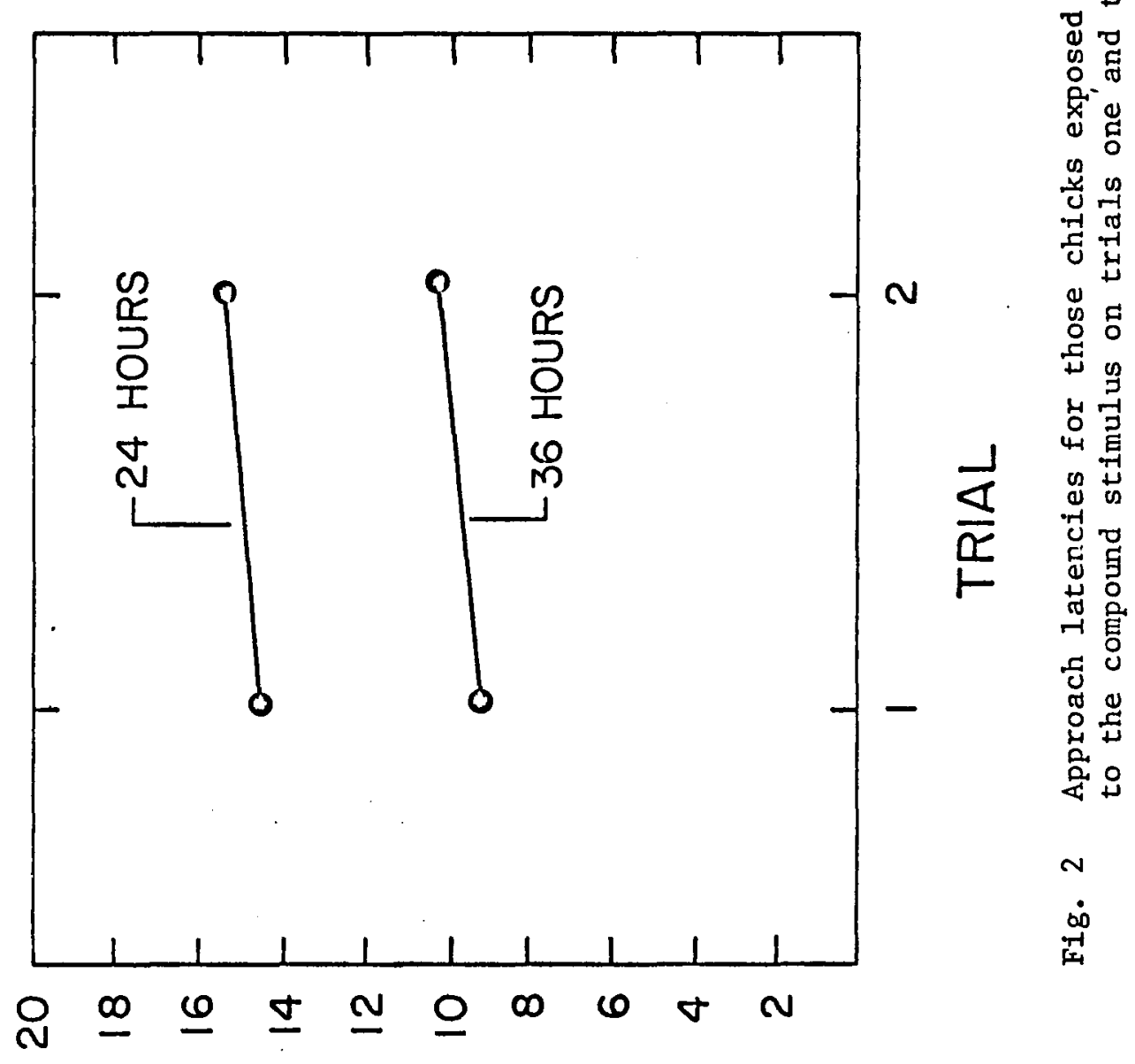

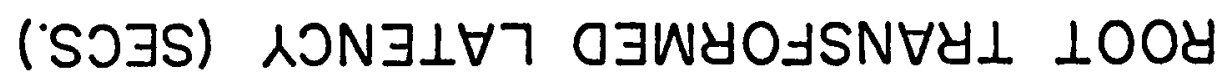




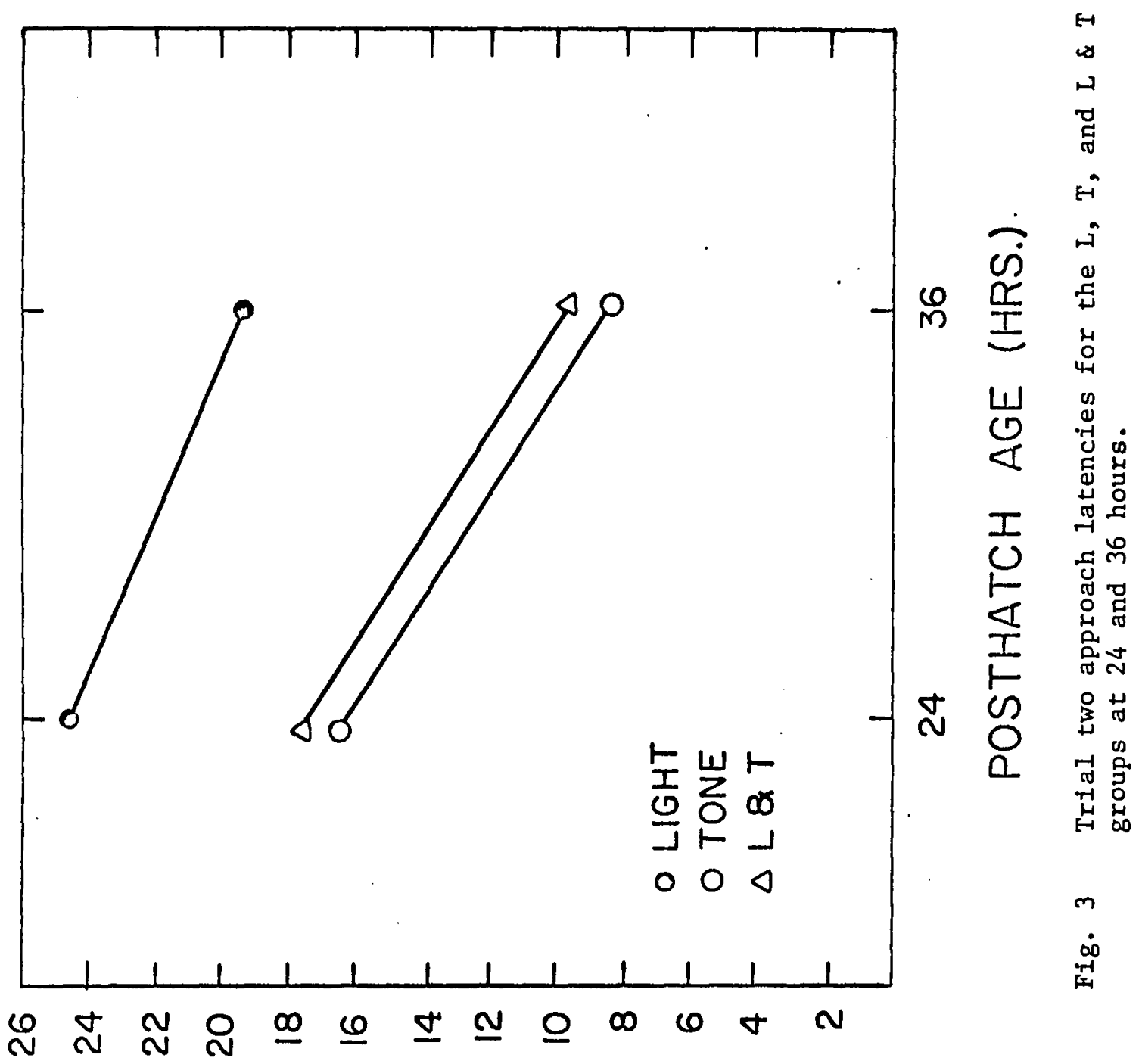

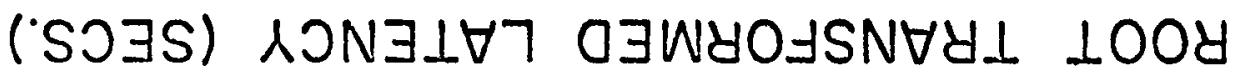


36 hours $(U=21, p<.05)$. Thus, the significant conditions effect is due to the overall longer latency for the $I$ group. There was no significant Age $x$ Condition interaction $\left(x^{2}<1\right)$.

As was noted above, many of the subjects did not approach on trial two, especially those subjects in the $L$ condition. Because the reason that some chicks failed to approach on trial two is unknown, an analysis of only those subjects which responded on trial was undertaken. Mean transformed latency scores for those subjects on trial two are agaln presented in Figure 4. Mean latencies are presented at both ages for the $T$ group $(24 \mathrm{hr} ., \mathrm{n}=8 ; 36 \mathrm{hr} ., \mathrm{n}=9)$, the L\& T group ( $24 \mathrm{hr} ., \mathrm{n}=8 ; 36$ hr., $n=8)$ and the $L$ group (24 hr., $n=2 ; 36 \mathrm{hr},, \mathrm{n}=5)$. The number of approachers in each group is also indicated on the figure. Analysis of Variance revealed a significant Age $x$ Condition interaction $(F=75.54$, $d f=2 / 34, p<.01)$. As can be seen in the figure, at 24 hours the groups do not differ, but the $\mathrm{L}$ group does show a significantly longer latency to approach at 36 hours, as determined by Duncan's Multiple Range test. The significant age-dependent decrease in latency to approach is again evident in the $T$ and the $L \& T$ condition $(F=14.42, d f=1 / 34, p<.01)$. The number of subjects approaching at both ages in the $T$ condition, $L$, and $L \& T$ conditions is presented in Figure 5. It is evident from the figure that the $T$ and $L \& T$ do not differ in attractiveness. In the L condition, however, a much larger number of subjects did not approach, though the figure does show a slight increase in number of approachers at 36 hours. A partitioning degrees of freedom $X^{2}$ (Winer, 1962, p. 629) comparing all three conditions, collapsed across age and again across conditions, revealed no significant differences, however, either 


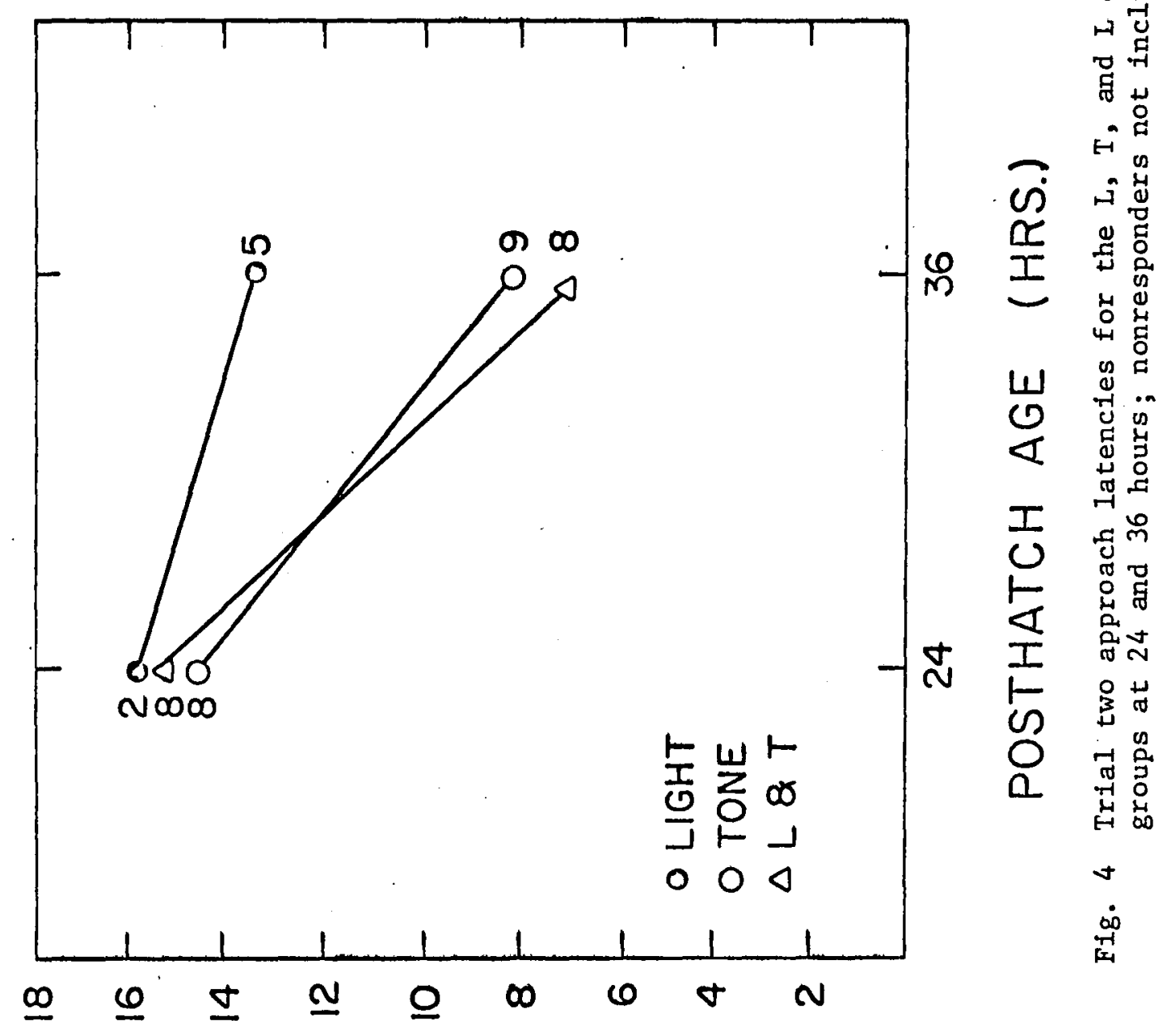

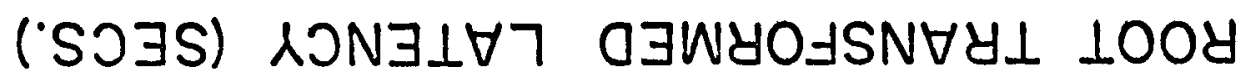




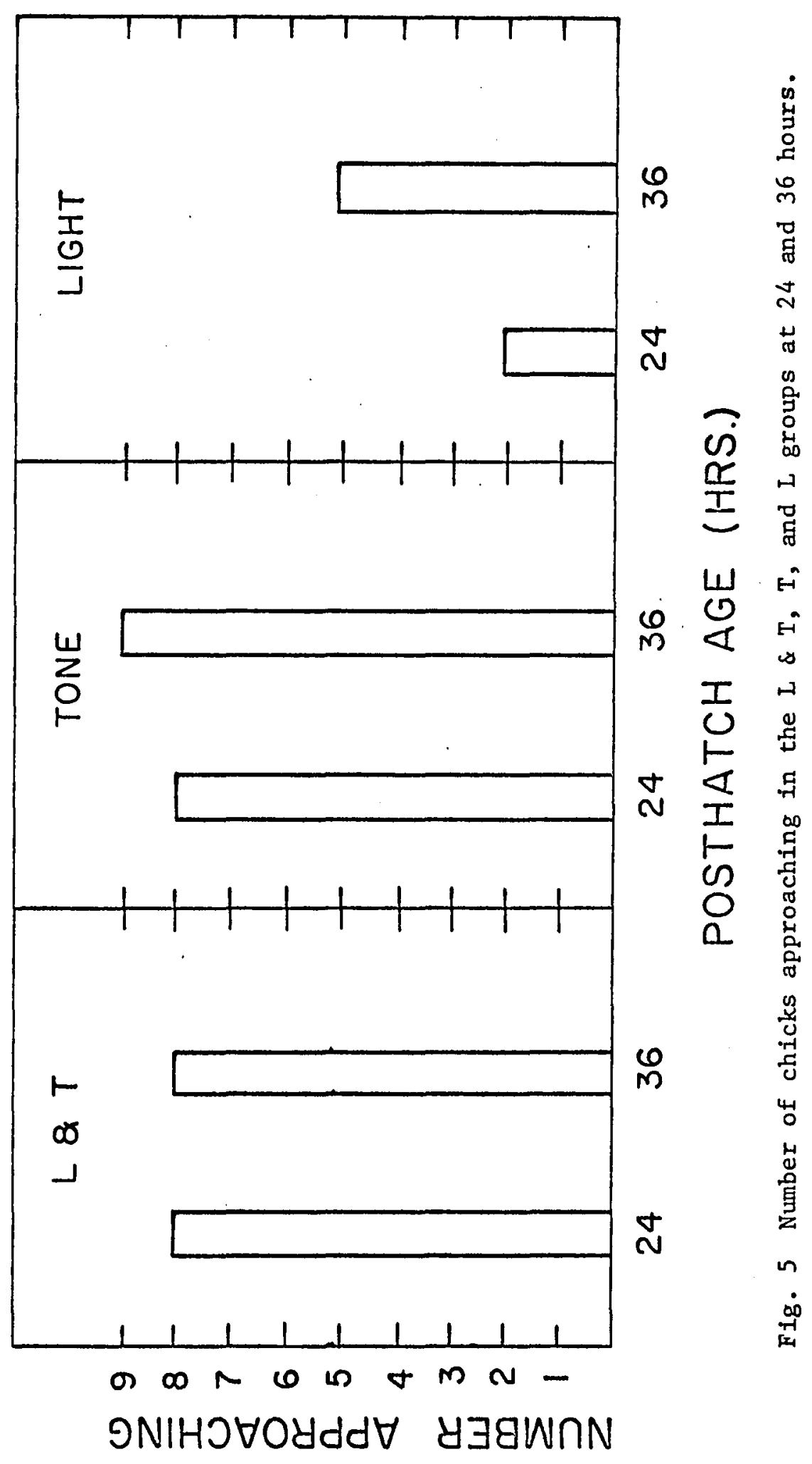


in age $\left(x^{2}<1\right)$, stimulus condition $\left(x^{2}=4.01, \mathrm{df}=2, \mathrm{p}>.05\right)$ or in the Age $x$ Condition Interaction $\left(X^{2}=1.94, \mathrm{df}=1, \mathrm{p}>.05\right)$. These results are not surprising in view of the nature of the data and the nature of the $x^{2}$ test. As degrees of freedom for $x^{2}$ increase, the obtained value must become proportionally larger in order to achleve significance. Since two of the conditions ( $L$ and $L \& T$ ) differ only by one subject, the effect of analyzing all three groups simultaneously is to increase degrees of freedom without substantially raising the obtained $\chi^{2}$ value. For this reason, the same $\chi^{2}$ analysis was performed on the $T$ $L$ groups, and again on the $L-L \& T$ groups. No analysis of the $T-L \&$ $\mathrm{T}$ groups was undertaken because, as was stated above, the graph reveals no great differences in the two. Analysis revealed that, in the $L-T$ comparison, the number of tone responders is significantly greater than the number of light responders $\left(X^{2}=4.08, \mathrm{df}=1, \mathrm{p}<.05\right)$. No age differences were revealed $\left(\chi^{2}<1\right)$ nor was any significant interaction obtained $\left(X^{2}<1\right)$. However, even though the $T$ and the $L \& T$ groups differ by only one subject, at 36 hours, the analysis of the L - L \& T groups revealed no significant stimulus condition differences $\left(X^{2}=3.62, \mathrm{df}=1\right.$, $\mathrm{p}>.05)$, no age differences $\left(X^{2}<1\right)$ and no interaction $\left(x^{2}<1\right)$.

\section{Following data}

Results simflar to those obtained with the approach data were obtained when the number of subjects following in each condition was examined (Figure 6). The $T$ and $L$ \& $T$ condition were found not to differ significantly $\left(X^{2}<1\right)$ and responding in the L condition was suppressed. A partitioning degrees of freedom $\chi^{2}$ of all three groups revealed, 


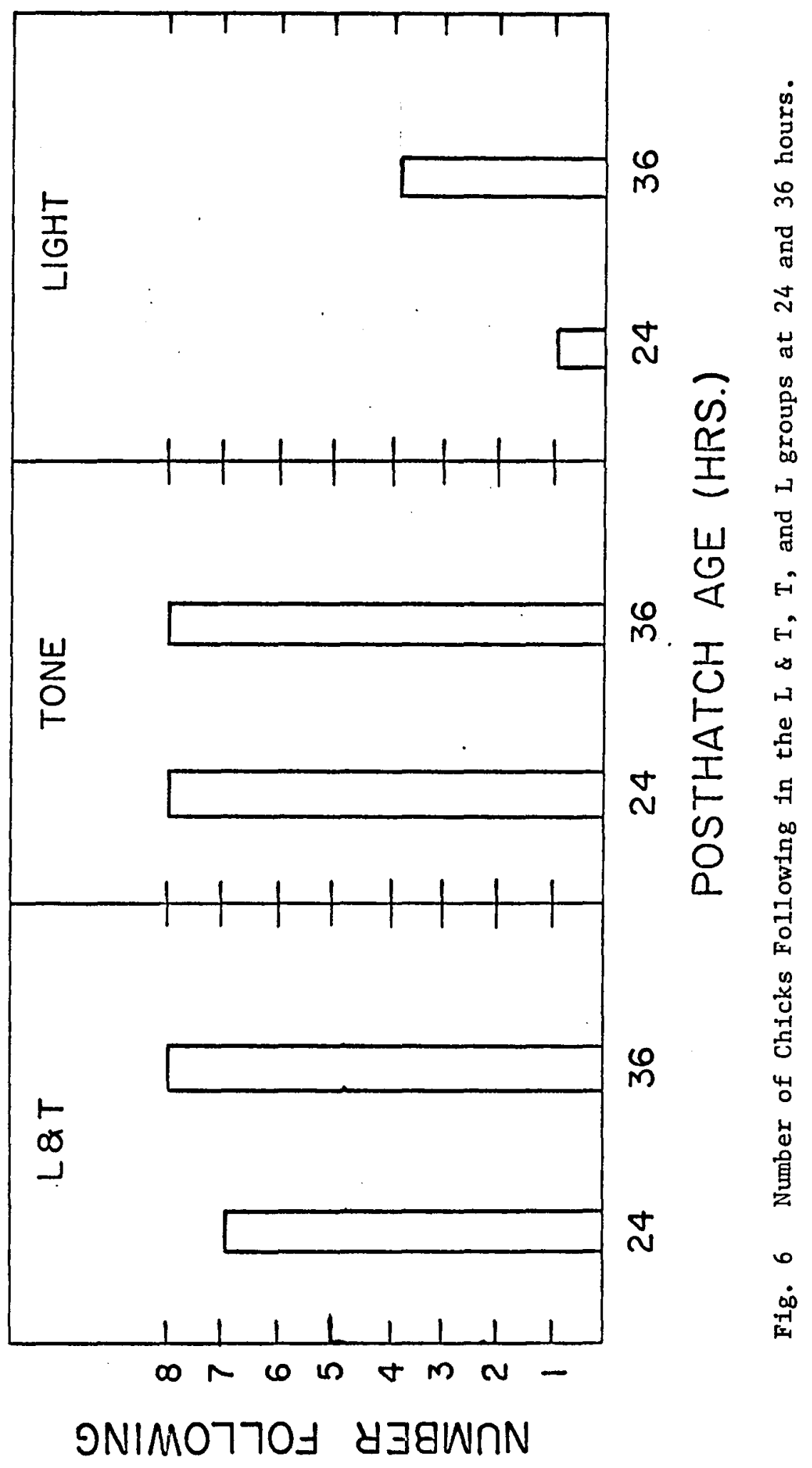


as was the case with the approach data, no signiflcant difference or interactions (overall $x^{2}=6.96, d f=5, p>.05$ ). Separate analysis of the $T$ - L conditions did reveal a significant difference in conditions $\left(\chi^{2}=5.76, \mathrm{df}=1, \mathrm{p}<.02\right)$; namely, significantly more subjects approaching the tone than the 1ight. Again, the slight increase in responding to $L$ at 36 hours is not significant $\left(x^{2}<1\right)$, and there was no significant Age $x$ Condition interaction $\left(\chi^{2}<1\right)$. Separate analysis of the L L \& T groups also revealed the same significant condition difference $\left(\chi^{2}=5, \mathrm{df}=1, \mathrm{p}<.05\right)$ and no age differences $\left(\chi^{2}<1\right)$ or Interaction $\left(x^{2}<1\right)$

The number of subjects following the light and the number of subjects following the tone in the $L$ vs. T condition is depicted in Figure 7. It appears that these data are in accord with the results of the $L$ and $T$ data: the tone is prepotent over the light at both ages and the slight trend for the number of responders to the light at 36 hours to increase is evident. A $X^{2}$ analysis confirmed that there are no age differences in number of followers of the tone, or the light $\left(\chi^{2}<1\right)$.

The number of light followers in the $L$ and the $L \underline{\text { vg. }} T$ condition is depicted in Figure 8 , as is the number of tone followers in $T$ and the $L$ vs. T condition. Fisher's Exact Probability tests revealed no significant differences in the number of light responders in the $L$ condition and the $L$ vs. $T$ condition, nor were any significant differences found in the number of tone followers in the $T$ and $L$ vs. $T$ conditions.

Returning to the results depicted in Figure 7 (L vs. T condition), It is possible to analyze these data, unlike the data obtained from the light only and tone only conditions, with a binomial test and the 


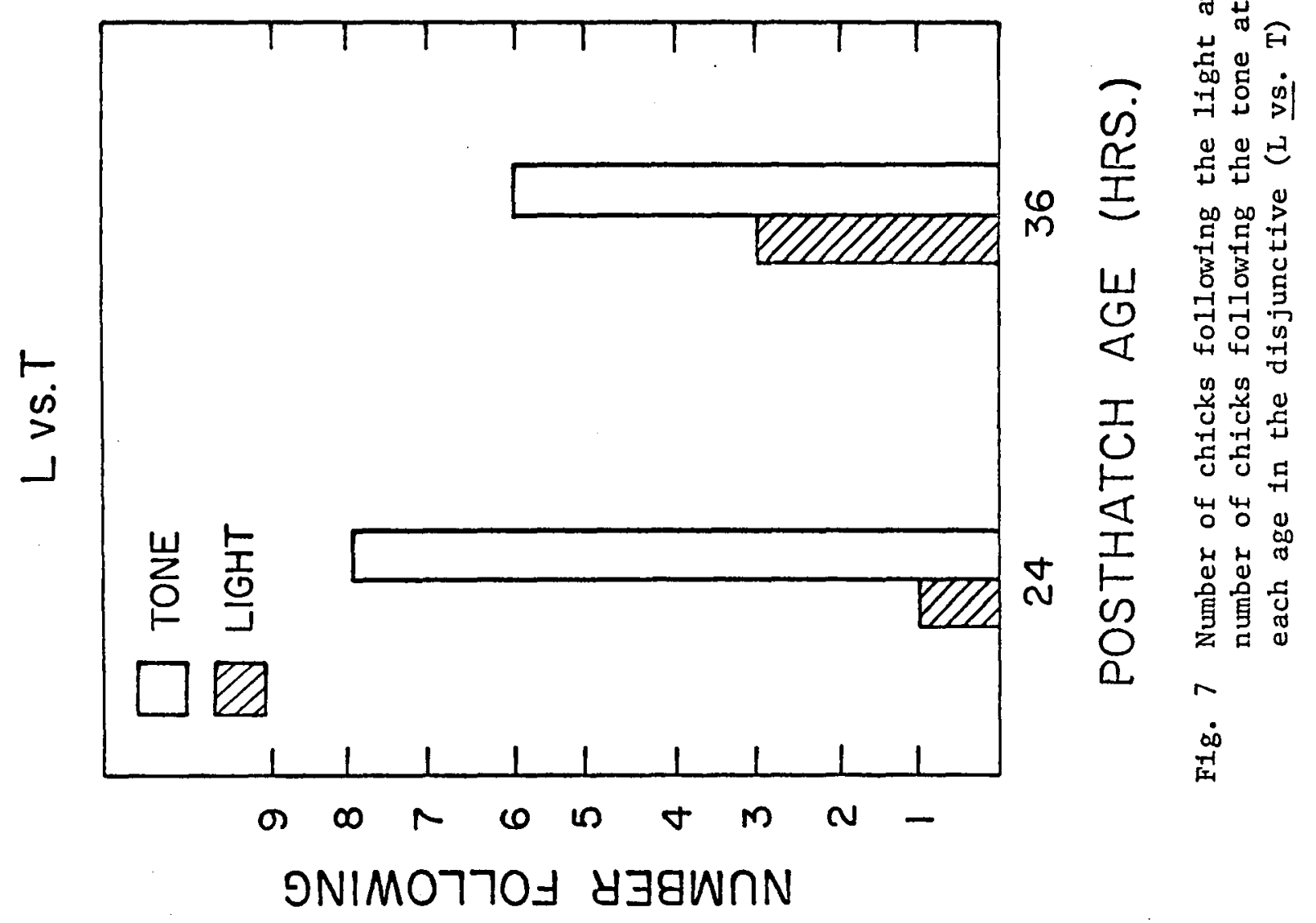




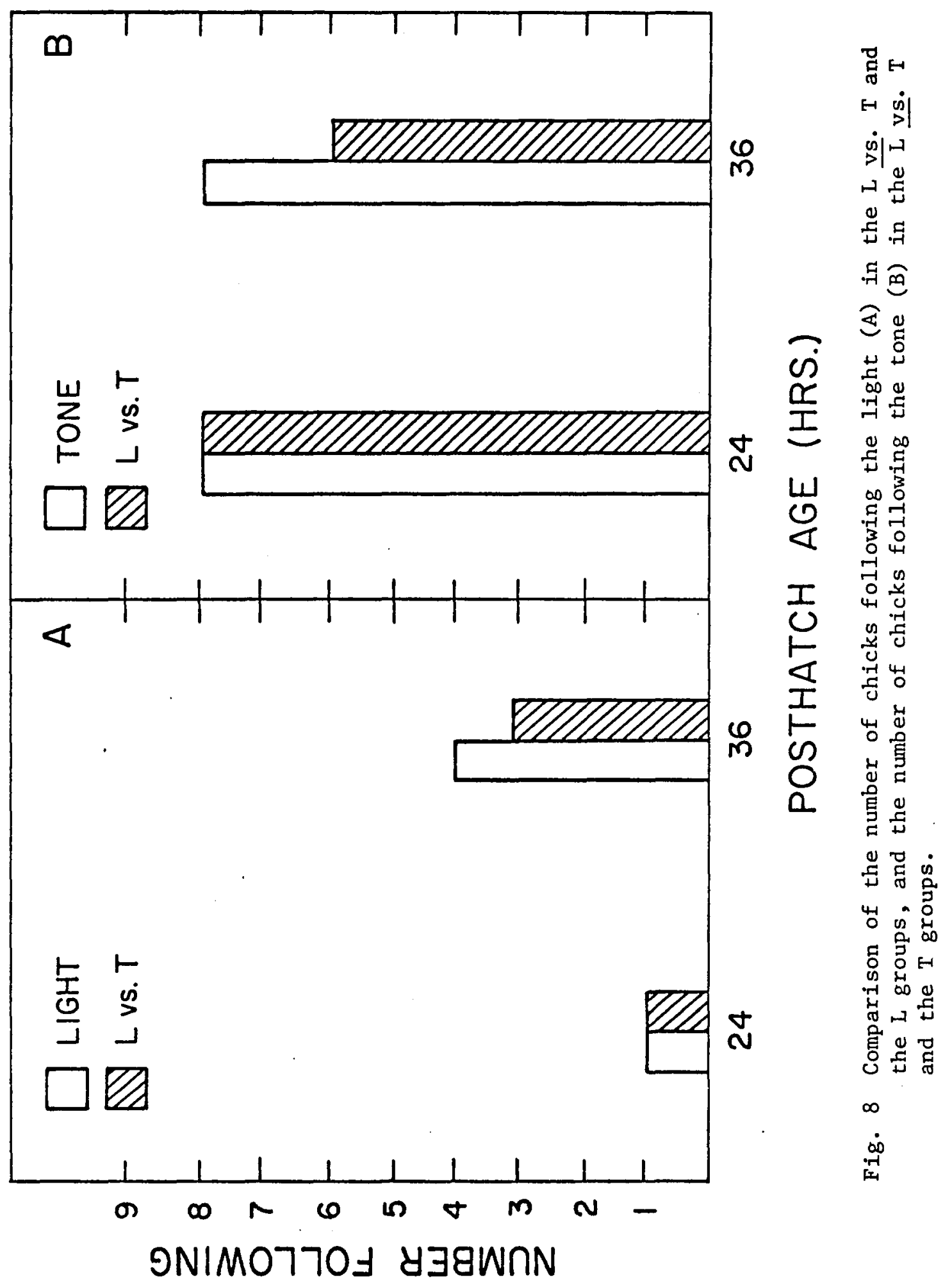


possibility of a slightly different interpretation of these data is suggested. If the null hypothesis (no differences in number of responses to tone or light) is accepted, the probability of following either stimulus is .5. At 24 hours, then, the binomial probability of one responder to the light and eight to the tone is .02 , indicating a significant preference for the tone. At 36 hours, the probability of $\mathrm{s} 1 \mathrm{x}$ tone responders and three light responders is .25 , indicating no signiflcant preference for either stimulus. 


\section{DISCUSSION}

The superfority of the tone over the 11ght at both ages tested is consistent with previous reports that auditory stimuli are prepotent over visual stimuli during the first few days after hatching (Fischer, 1966; Gottlieb, 1963; Gottlieb \& Simner, 1969). The age-dependent increase in responsiveness is also consistent with previous reports, both for auditory stimuli (Gottlieb, 1963) and visual stimuli (Gottlieb, 1963; Kovach, Paden \& Wilson, 1968). However, contrary to previous reports (Gottlieb, 1968; Gottlieb \& Simer, 1969) the compound stimulus was not found to be significantly more effective in elfciting approach and following responses than either of the component stimuli alone; virtually all measures in the present study reflect no difference in the attractiveness of the compound auditory-plus-visual stimulus and the auditory stimulus alone.

The relative ineffectiveness of the visual stimulus employed in the present study as a releaser of the approach response is reflected in the longer latency to respond to just the visual stimulus at both ages tested, as well as the small numbers of subjects at each age which approached just the visual stimulus. Further evidence of the relative Ineffectiveness of the visual stimulus is found in the latency scores of those subjects which did approach the visual stimulus on trial two. These data suggest that the longer latency to approach is due to the number of nonresponders which were given maxtmum approach scores. At 24 hours, if nonresponders are not included, there are no differences in latency to approach; the true difference between the stimulus conditions 
lies in the number of subjects approaching each stimulus. In going from 24 to 36 hours of age, however, there is a decreased latency to approach the auditory stimulus and the auditory-plus-visual stimulus; the decreased latency to approach with age is not found in the light only condition. Thus, the chicks are elther responding, at 24 hours, to the light at near minimal latency times, or the visual stimulus is not sufficiently attractive to Induce the 36 hour old birds into responding with a shorter latency. Of course, the fact that the 36 hour chicks approached the tone only and the L \& $T$ stimull significantly sooner than older chicks in the light only condition indicates that the latter is the case.

The Interpretation of the inverse relationship between age and latency to approach is consistant with at least two hypotheses. One is that the older chicks are stronger and are able to leave the start box and carry themselves towards the stimuli more rapidly than the younger, weaker 24 hour chicks. The other interpretation is that with age the chicks are more susceptible to the releasing properties of the auditory stimuli. The fact that age alone does not necessarily produce a decrease in latency to approach a particular stimulus, as was the case with L above, gives support to the second of these hypotheses.

The apparent superiority of the auditory over the visual stimulus is again clearly demonstrated by the following response data. It is evident that chicks are more responsive to auditory than visual stimuli at the ages tested and that the visual stimulus is, again, relatively ineffective as a releaser of the response. Unllke the approach data, however, no significant age differences in the attractiveness of any of 
the stimuli is evident. This probably reflects, at least in the case of the auditory stimulus and the auditory-plus-visual stimulus, the relative insensitivity of the following measure; at 24 hours the auditory stimulus is sufficiently potent to elicit asymptotic response levels as reflected in the following measure, and therefore no change occurred with time. There is no evidence of any behavioral polymorphism (Klopfer \& Gottlieb, 1962). In the responses of the chicks; the very slight Increase with age in following the visual stimulus is not statistically significant and the attractiveness of the visual stimulus does not approach that of the tone.

The following data obtained from the disjunctive analysis (light vs. tone) are consistant in most respects with those obtained in the more traditional simultaneous choice conditions. The very small agedependent Increase In responsiveness to the light may be associated with, because of the nature of the procedure, a very small age-dependent decrease in responsiveness to the tone, but nelther are statistically reliable. Even though at 24 hours the chickens show a marked preference for the visual stimulus and at 36 hours this preference, while still evident, has decreased, the evidence that a preference for the visual stimulus over the auditory stimulus develops with age, as a function of the maturation of the chick's visual system (Gottlieb \& Klopfer, 1962) must be regarded as merely suggestive. The small number of subjects whlch experlenced the disfunctive procedure provides results which are not sufficiently convincing to override the consistant lack of significant age-dependent shifts in attractiveness of the stimuli which was found repeatedly in this study. A more sensitive instrument than the 
relatively crude follow-not follow measure may reveal, in future studies, more rellable differences. For present purposes, however, it must be concluded that, at least for the first 36 hours of life, the disfunctive analysis is in agreement with the more traditional analysis procedure in indicating that chicks respond primarily to auditory stimuli upon initial exposure to the stimulus.

Such a preference for the conditions in which the auditory stimulus was present (as indicated by the fact that the $T$ and $L \& T$ groups $d 1 d$ not differ in any of the measures) suggests the operation of a type of stimulus control referred to in the learning 1iterature as overshadowing. Overshadowing is a well-known result of some learning studies Involving compound stimulus conditioning. It is frequently reported that, when a response is conditioned in the presence of a compound stimulus, subsequent analysis of the component stimuli alone reveals that the response has become assoclated with the more prominent of the components. The prominent feature of the compound stimulus overshadows the less prominent features. It is assumed, if not actually proven in subsequent trials, that it is possible to differentially condition the response to either of the component stimull alone (M1les, 1965). The present situation differs from that of the overshadowing situation in that the response in this situation is not learned (Sluckin, 1973, p. 8). The results obtained from the present study have an application which was rather unexpected, and that conterns the role of auditory stimul1 in 1mprinting. Gottlieb (1968) has suggested that auditory stimull may possess a general orlenting or attention-directing function, whereas Pitz \& Ross (1961) suggest that auditory stimuli in the 
imprinting situation may possess a general arousal function stemming from the presence of the sound. Fischer (1966) has reported two functlons of the auditory stimulus: 1) a general arousal function and 2) a specific-recognition function. The percentages of chicks approaching each stimulus condition bear on these hypotheses. As will be recalled, at 24 hours and 36 hours of age, on trial one the approach rate is much higher than that of the pilot data. Results of the present study indicate that this is not due to a significant increase in the attractiveness of the light, but rather of an Increase of attractiveness of the tone. It appears, then, that the tone does not possess by itself a general arousal function, or a greater responsiveness to the light only on the next trial would result. Similarly, an attention-directing hypothesis would predict a greater responsiveness to the light only following 5 minutes exposure to both stimull emanating from a common source. Only the specific-recognttion function (F1scher, 1966) can adequately explain these results. If the tone serves a recognition function, then the presentation of the light without the accompanying tone should not produce responding to any significant degree, which it did not. 


\section{Summary \& Conclusions}

An attempt was made to assess the relative Importance of auditory and visual stimuli in initiating approach and following responses in neonatal chicks during the inftial exposure to compound stimuli. TestIng, which was done 24 and 36 hours posthatch, was accomplished by exposing subjects to a compound auditory-plus-visual stimulus and then measuring responsiveness to the component stimuli (a pulsating tone and a flickering light) alone, and by measuring following responses to a disfunction of the compound stimulus. Conclusions were:

1) Neonatal chicks respond (by approach and following) primarily to auditory stimuli during the first 36 hours of $11 f e$.

2) There are no significant changes with age in this stimulus preference.

3) There are no significant differences in the results obtained by the two test procedures employed.

4) Some evidence that auditory stimuli have a specific recognition function was obtained. 


\section{LITERATURE CITED}

Boyd, H. \& Fabricius, E. Observations on the Incidence of following of visual and auditory stimuli in naive mallard ducklings (anas platyrhnchos). Behavior, 1965, 25, 1-15.

Collias, N. E. The development of social behavior in birds. Auk, 1952, $69,127-159$.

Collias, N. E. \& Collias, E. C. Some mechanisms of family Integration in ducks. Auk, 1956, $\underline{73}, 378-400$.

Fabricius, Eric. Some experiments on imprinting phenomena in ducks. Proceedings of the Tenth International Ornithological Congress, 1951, 375-379. Cited by E. H. Hess, Imprinting, New York:

D. Van Nostrand, 1973, Chapter 3.

Fabricius, Eric. Experiments on the following-response of mallard ducklings. British Journal of Anlmal Behaviour, 1955, 3, 122.

Fabricius, E. \& Boyd, H. Experiments on the following reactions of wild ducklings. Wildfowl Trust Fund Annual Report, Slimbridge, England, 1954, 6, 84-89. Cited by E. H. Hess, Imprinting.

New York: D. Van Nostrand, 1973, Chapter 3.

Fischer, G. J. Auditory stimull in imprinting. Journal of Comparative and Physiological Psychology, 1966, 61, 271-273.

Fischer, G. J. Sound stimuli and following in a domestic fowl: Frequency, rate, and duration. Journal of Comparative and Physiological Psychology, 1972, 81, 2, 183-190.

Gottlieb, G. Following-response initiation in ducklings: Age and sensory stimulation. Science, 1963, 140, 399-400.

Gottlieb, G. The question of imprinting in relation to parental and species identiflcation by avian neonates. Journal of Comparative and Physiological Psychology, 1965, 59, 345-356.

Gottlieb, G. Species identification by avian neonates: Contributory effect of perinatal auditory stimulation. Animal Behaviour, 1966 , 14, 282-290.

Gottlieb, G. Species recognition in ground-nesting and hole-nesting ducklings. Ecology, 1968, 49, 282-290.

Gottlieb, G. \& Klopfer, P. H. The relation of developmental age to auditory and visual imprinting. Journal of Comparative and Physiological Psychology, $1962,55,5,821-826$. 
Gottlieb, G. \& Simner, M. L. Auditory versus visual flicker in directing the approach response of domestic chicks. Journal of Comparative and Physiological Psychology, 1969, 67, 58-63.

Gray, P. H. Evidence that retinal flicker is not a necessary condition of imprinting. Science, 1960, 132, 1834-1835.

Hess, E. H. Imprinting: Early experience and the development psychobiology of attachment. New York: D. Van Nostrand, 1973.

Hinde, R. A., Thorpe, W. H. \& Vince, M. A. The following response in young coots and moorhens. Behaviour, 1956, 9, 214-242.

James, H. Flicker: an unconditioned stimulus for imprinting. Canadian Journal of Psychology, 1959, 13, 2, 59-67.

James, H. Imprinting with visual flicker: Evidence for a critical period. Canadian Journal of Psychology, 1960, 14, 1, 13-19.

Jaynes, J. Imprinting: the interaction of learned and innate behavior: II. The critical period. Journal of Comparative and Physiological Psychology, 1957, 50, 6-10.

Klopfer, P. H. An analysis of learning in young anatidae. Ecology, $1959, \underline{40}, 90-102$.

Klopfer, P. H. \& Gottlieb, G. Imprinting and behavioral polymorphism: Auditory and visual imprinting in domestic ducks (anas platyrhynchos) and the involvement of the critical period. Journal of Comparative and Physiological Psychology, 1962, 55, 126-130.

Kovach, J. K. Critical period of optimal arousal? Early approach as a function of stimulus, age, and breed variables in chicks. Developmental Psychology, 1970, 3, 73-77.

Kovach, J. K. Effectiveness of different colors in the elicitation and development of approach behaviour in chicks. Behaviour, 1971 (a), 38, 154-168.

Kovach, J. K. Interaction of Innate and acquired: Color preferences and early exposure learning in chicks. Journal of Comparative and Physiological Psychology, 1971 (b), $\underline{75}, 386-398$.

Kovach, J. K. \& Hickox, J. E. Color preferences and early perceptual discrimination learning in domestic chicks. Developmental Psychobiology, 1971, 4 , 255-267.

Kovach, J. K., Paden, P. \& Wilson, G. Stimulus variables in the elicitation and short-range reversibility of approach and following responses. Journal of Comparative and Physiological Psychology, $1968,66,175-178$. 
Lorenz, K. (1935). Companionship in bird life; fellow members of the species as releasers of social behavior. In Schiller, C. H. (Ed.), Instinctive Behavior. New York: International University Press, 1957.

Lorenz, K. (1937). The nature of instinct. In Schiller, C. H. (Ed.), Instinctive Behavior. New York: International University Press, 1957.

Miles, C. G. Acquisition of control by the features of a compound stimulus in discriminative operant conditioning. Unpublished doctoral dissertation, McMaster University, 1965.

Moltz, H. Imprinting: Empirical basis and theoretical significance. Psychological Bulletin, 1960, 57, 4, 291-314.

Moltz, H. Imprinting: an epigenetic approach. Psychological Review, $1963, \underline{70}, 123-138$.

Pitz, G. F. \& Ross, R. B. Imprinting as a function of arousal. Journal of Comparative and Physiological Psychology, 1961, 54, 602-604.

Ramsey, A. 0. \& Hess, E. H. A laboratory approach to the study of imprinting. Wilson Bulletin, $1954,66,3,196-205$.

Salzen, E. A. \& Sluckin, W. The incidence of the following response of domestic fow1. Animal Behaviour, 1959, 7, 172-179.

Schulman, A. H., Hale, E. B. \& Graves, H. B. Visual stimulus characteristics for initial approach response in chicks. Animal Behaviour, $1970,18,461-466$.

Simner, M. L. Cardiac self-stimulation hypothesis and the response to visual flicker in newly hatched chicks: Preliminary findings. Proceedings of the $74 \mathrm{th}$ Annual Convention of the American Psychological Association, 1966, 1, 141-142. Cited in Hess, E. H., Imprinting. New York: D. Van Nostrand, 1973.

Sluckin, W. Imprinting and Early Learning. ChIcago: Aldin, 1973

Smith, F. V. Towards definition of the stimulus situation for the approach response in the domestic chick. Animal Behaviour, 1960, 8, 3-4, 197-200.

Smith, F. V. \& Bird, M. W. The relative attraction for the domestic chick of combinations of stimuli in different sensory modalities. Anima1 Behaviour, 1963, 11, 300-305.

Smith, F. V. \& Hoyse, P. A. Properties of the visual stimuli for the approach response in the domestic chick. Animal Behaviour, 1961, 9, 3-4, 159-165. 
Spalding, D. A. Instinct, with original observations on young animals. McMillan's Magazine, 1873, 27, 282-293. Reprinted in British Journal of Animal Behaviour, $1954,2,2-11$.

Wilson, K. V. A distribution-free test of analysis of variance hypotheses. Psychological Bulletin, 1956, 56, 1, 96-101.

Winer, B. J. Statistical Principles in Experimental Design. New York; McGraw-Hill, 1962. 


\section{The vita has been removed from the scanned document}




\title{
A DISJUNCTIVE ANALYSIS OF NEONATAL APPROACH STIMULUS PRETOTENCE
}

by

\author{
Stephen E. Sigman
}

\begin{abstract}
(ABSTRACT)
A disjunctive analysis technique was developed to investigate the relative Importance of auditory and visual stimulf in eliciting inftial approach and following responses in domestic fowl. 72 Canadian Athens random bred chicks were tested at 24 or 36 hours posthatch. A repetitive, tapping tone ( 4 per sec., 50 msec. duration, $500 \mathrm{~Hz}$ ) served as the auditory stimulus, and a flickering light ( $3.5 \mathrm{pps}, .8 \mathrm{fcl}$. Intensity) served as the visual stimulus. Results are in agreement with previous reports that chicks of the ages tested respond primarily to auditory stimuli. However, a nonsignificant trend for an age-dependent increase in attractiveness of visual stimuli, with a concomitant decrease in attractiveness of auditory stimuli, was found.
\end{abstract}

